\title{
The Optimal Suture Technique for Mitral Valve Replacement in an Ex Vivo Animal Model
}

\author{
Emilie Schultz Hougaard, Marie Schäfer, Lars Peter Riber \\ Department of Cardiac, Thoracic and Vascular Surgery at Odense University Hospital, Odense, Denmark \\ Email: emilieschultzh@gmail.com, marie_schafer11@hotmail.com, lars.riber@rsyd.dk
}

How to cite this paper: Hougaard, E.S., Schäfer, M. and Riber, L.P. (2021) The Optimal Suture Technique for Mitral Valve Replacement in an Ex Vivo Animal Model. Open Journal of Thoracic Surgery, 11, 36-45. https://doi.org/10.4236/ojts.2021.111006

Received: February 8, 2021

Accepted: March 21, 2021

Published: March 24, 2021

Copyright $\odot 2021$ by author(s) and Scientific Research Publishing Inc. This work is licensed under the Creative Commons Attribution International License (CC BY 4.0).

http://creativecommons.org/licenses/by/4.0/

\begin{abstract}
Objective: This study compared and evaluated three suture methods in order to find the method with the best durability for mitral valve replacement. The methods evaluated are horizontal mattress suture with subanular pledgets, horizontal mattress suture with supraanular pledgets and continuous running suture. Methods: Thirty hearts were explanted from newly terminated pigs. The hearts were randomized in the three groups. After an atriotomy, the mitral valve was cut out, and a patch was sutured into its place. An air pump model was connected to a balloon which was placed in the left ventricle through aorta and continuously filled with air to a maximum of $300 \mathrm{mmHg}$. The peak pressure at rupture was noted. If no rupture occurred before reaching $300 \mathrm{mmHg}$, the suture was found competent. Results: Two out of ten hearts in the continuous running suture-group had myocardial rupture within a pressure of $300 \mathrm{mmHg}$. In the remaining eight hearts there were no ruptures within $300 \mathrm{mmHg}$. In the two groups sutured with horizontal mattress with pledgets placed either subanular or supraanular, no rupture of myocardium occurred. When comparing continuous running suture with the horizontal mattress with subanular pledgets or the horizontal mattress with supraanular pledgets, the 1 -sided Fishers' exact was 0.237 . At $5 \%$ significance level, there was no difference between the three suture methods. Conclusion: There was no statistically significant difference between the durability of the three suture methods, though rupture was only evident in the continuous running suture line. Since it is the surgeon's choice to select the optimal suture technique, our study should be a reminder for the surgeon to reflect on the suturing factors that have an influence on successful mitral valve replacement.
\end{abstract}

\section{Keywords}

Mitral Valve, Mitral Valve Replacement, Suture Technique 


\section{Introduction}

The estimated prevalence of all valve diseases in adults is $2.5 \%$, and the prevalence increases with age [1].

The preferred surgical treatment for non-ischemic mitral valvular regurgitation is repair of the original valve [2]. If the mitral valve is non-repairable due to severe calcification or extensive leaflet destruction, a replacement of the valve is preferable [2].

Although the 30-day mortality in 2019 in Denmark was zero in all isolated mitral valve replacements (MVR), [3] paraprosthetic leakage (PPL)/paravalvular leakage (PVL) is well-recognized complications of prosthetic valves especially in calcified mitral annuli, for which reason optimal surgery is necessary. The incidence of PPL/PVL detected by transesophageal echocardiography is up to $15 \%$ in the first postoperative period though small leaks in asymptomatic patients are found to be benign. These are followed by regular echocardiography to ensure that they do not worsen, in which case re-operation could be necessary [4]. Further, PPL/PVL is associated with increased risk of infection, annular calcification and left ventricular hypertrophy [5]. Most often, PPL/PVL is due to an incomplete suture line, but it can also occur due to rupture of the suture or due to the fact that the sutures have been pulled through the sutured tissue [5]. Thus, the durability of the sutures and the operation technique used are important factors in preventing or creating PVL and thereby the risk of need for surgical revision.

The fact that there is little or no tissue in growth into the prosthetic ring regardless of the age of the prosthetic valve [6], supports the hypothesis that an intact and durable suture line is important.

Only a few clinical studies regarding the optimal implantation of the mitral valve have been conducted.

1) No differences in the incidence of PVL have been found between pledgeted and non-pledgeted sutures [2].

2) No difference between the semicontinuous technique and interrupted suture techniques has been found, though the semicontinuous technique might be safer and more reliable due to shorter bypass time [7].

Newton et al. [8] did, however, find that

1) Suture techniques with pledgets were more durable compared to non supported sutures.

2) Horizontal mattress with subanular pledgets was more durable than horizontal mattress with supraanular pledgets in in vitro canine hearts.

Finally, Stiles et al. did not find any significant difference in durability between suture techniques with pledgets and non-supported sutures, though they tend to conclude that sutures with pledgets are superior [6].

Since evidence is so sparse, no optimal technique is yet established, and it relies on the surgeon to select the optimal suture technique. Therefore, the suture technique of a mitral valve replacement varies from surgeon to surgeon. 
In this study, we compare and evaluate the durability of three suture methods in 30 porcine hearts in order to find the optimal suture method for mitral valve replacement. The methods evaluated are the most commonly used suture techniques for MVR in Scandinavia: Horizontal mattress suture with subanular pledgets, horizontal mattress suture with supraanular pledgets and continuous running suture.

\section{Materials and Methods}

We examined 30 hearts from female pigs between the 27th of August and 9th of December at the Biomedical Laboratory at the University of Southern Denmark. 30 hearts were available during the study period, and this sample size matches that of the previous studies [6] [8] [9]. The pigs weighed 25 - $45 \mathrm{~kg}$, and we had no influence on the size of the pigs we received.

All of the porcine hearts were explanted from newly terminated pigs used for other biomedical research or surgical skills courses, which all had the needed approvals for their respective studies. Therefore, there were no further ethical considerations to be made. None of the hearts had been investigated nor harmed in their respective studies. If the tissue of left ventricle was damaged and thereby weakened during the explanation, it was excluded from the study. Two medical students performed the procedures on the hearts after training and supervision from a chief heart surgeon.

We received the pigs when they were still alive but sedated. Shortly hereafter they were terminated with Phenobabital (Fenemal) as planned. Just hereafter, we performed a median sternotomy and dissected the hearts out of the pigs. A minimum of $3 \mathrm{~cm}$ of the ascending aorta was preserved to ensure room for our air pump model. The remaining of the great vessels was dissected from the hearts.

The explanted hearts were then either operated on directly or cooled down until the procedure was performed the next morning.

The hearts were permuted block randomized and assigned to one of three groups with a six-sided dice, whereby 10 hearts were assigned to each group.

Within 24 hours of termination the mitral valve was exposed by an atriotomy of the explanted heart, and the mitral valve was excised near the anulus of the valve leaving a $2-4 \mathrm{~mm}$ rim of leaflet tissue. Instead of a prosthetic mitral valve we used a patch from a dacron aortic tube prothesis, since our economy did allow us to use 30 mitral valves.

The patch was cut to fit the size of the mitral annulus and then sutured into it with one of three methods (Figure 1):

1) Horizontal mattress suture with Ethibond 2-0 with polymer pledgets from the atrial side.

2) Horizontal mattress suture with Ethibond 2-0 with polymer pledgets from the ventricle side.

3) Continuous running suture with Prolene 3-0. 
A
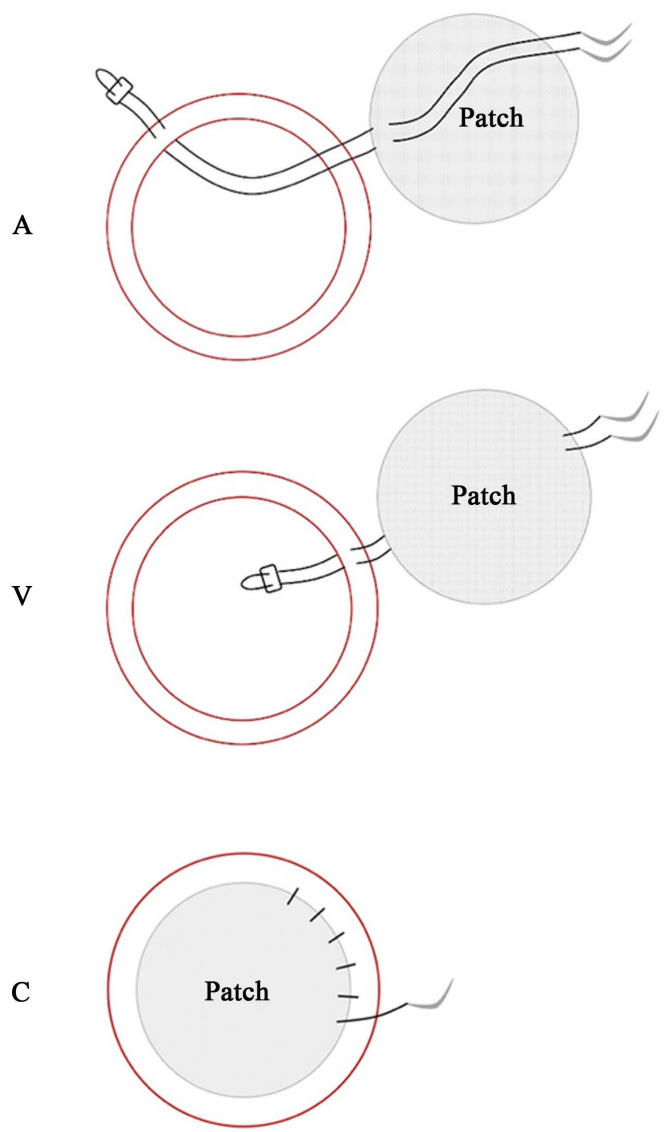

Figure 1. The three suture methods. The red ring represents the mitral annulus. A: Horizontal mattress suture with suppranular pledgets. V: Horizontal mattress suture with subanular pledgets. C: Continuous running suture.

The suture line was carefully placed in the fibrous ring of the mitral anulus. The size of bites and annular depth was kept constant matching normal procedure.

The aortic valve was hereafter partially removed in order to measure the pressure in the left ventricle.

A canular needle was inserted into a balloon which was then inserted through the aortic root and placed in the left ventricle (Figures 2-4). A Philips IntelliVue $\mathrm{X} 3$ patient monitor was connected to the canular needle in order to measure the pressure. After this an air pump was connected to the balloon. The canular needle was secured in the aortic root with cable ties to hold it in place and prevent air leakage.

The pressure was increased progressively by pumping air into the balloon until rupture of either the suture or tissue was observed, which was considered the endpoint. The pressure was only applied for a couple of seconds simulating the normal physiological conditions of human heartbeat. If no rupture of suture or tissue occurred at $300 \mathrm{mmHg}$, no further pressure was applied, and the suture was found competent since a pressure above $300 \mathrm{mmHg}$ is assumed not to be relevant in a human being. 


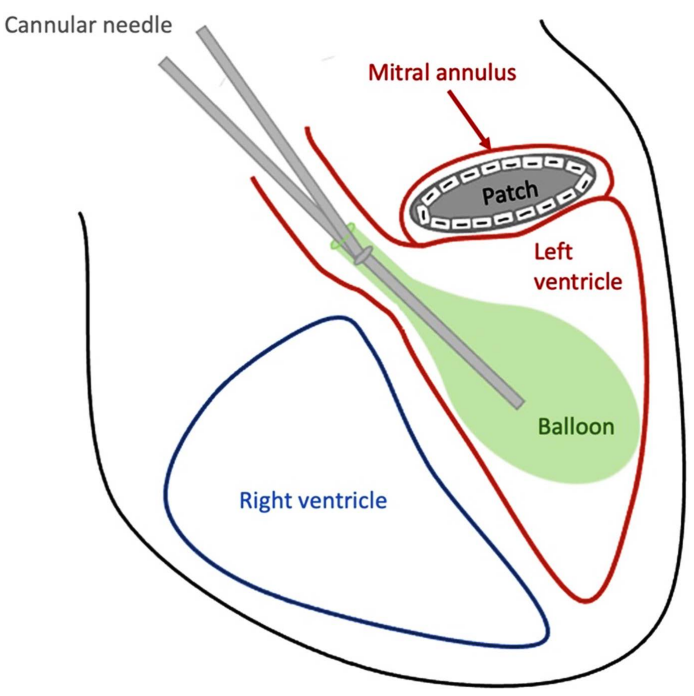

Figure 2. The experimental setup. The explanted porcine heart model with the balloon placed in the left ventricle through the aorta. Within the balloon is a canular needle dividing into two tubes. One of the tubes is connected to the air pump and the other tube is connected to the monitor measuring the pressure.

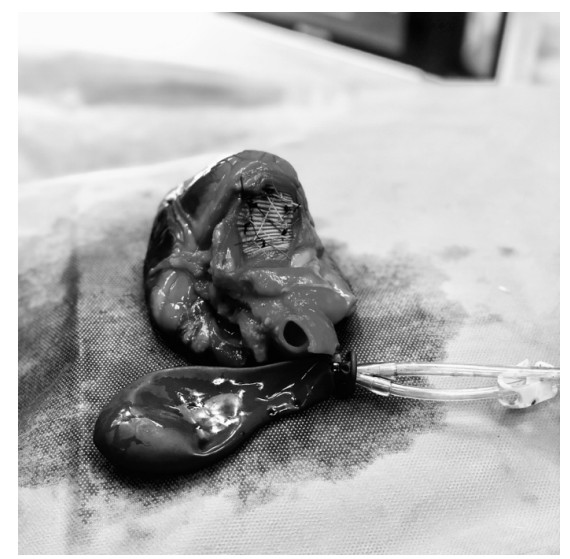

Figure 3. The explanted heart after atriotomy and a patch sutured in the mitral anulus. In front of the heart lies the balloon with the canular needle inside.

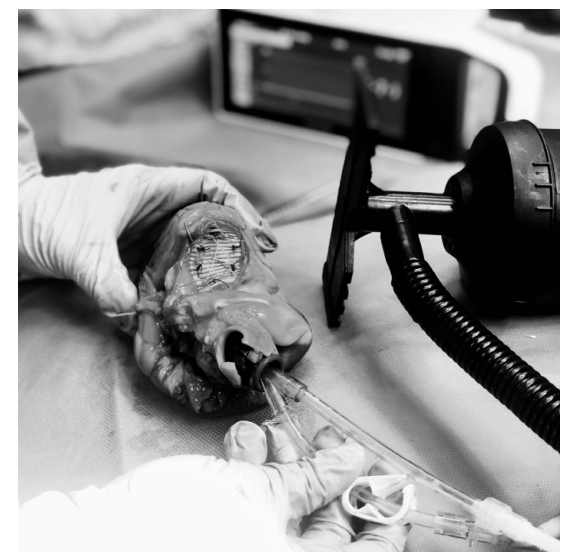

Figure 4. The explanted heart with the balloon placed in the left ventricle through the aorta. The monitor and the air pump are visible in the background. 
Blinding of the surgeon was not an option, but to minimize bias and ensure quality, the hearts were approved at three different checkpoints by the other investigator who did not perform the procedure on the given heart. The checkpoints were:

1) Ensuring the $2-4 \mathrm{~mm}$ rim of leaflet tissue left in place after atriotomy and excision of the valve.

2) Accepting the size of the patch after cutting it.

3) Finally, approving the suture line and potential pledgets and their placement before pressure test.

Data from the pressure test was collected by the two investigators and analyzed using Fisher's exact 1-sided test (p-value $<0.05$ ) and STATA 16.0.

\section{Results}

Myocardial ruptures occurred in two out of ten hearts in the continuous running suture-group before reaching a pressure of $300 \mathrm{mmHg}$ (Table 1). In both hearts myocardial rupture occurred in the posterior portion of the mitral anulus. In the other eight hearts in this group there were no ruptures of the myocardial tissue before reaching a pressure $300 \mathrm{mmHg}$.

No rupture of the myocardial tissue occurred in the two other groups sutured with horizontal mattress with pledgets placed either subanular or supraanular (Table 1).

The sutures and patches remained intact in all 30 hearts of the study.

When comparing continuous running suture with the horizontal mattress with subanular pledgets or the horizontal mattress with supraanular pledgets the 1-sided Fishers' exact was 0.237 .

At $5 \%$ significance level there was no statistically significant difference between the durability of the three suture methods.

\section{Discussion}

In two of the hearts, the myocardial tissue ruptured in between the atrium and ventricle close to the suture line at respectively $270 \mathrm{mmHg}$ and $300 \mathrm{mmHg}$. Our findings suggest that the continuous running suture line had weakened the myocardial tissue and caused the rupture. The rupture was only found in smaller, younger pigs with less muscular heart tissue. The weight of the pig might be a contributing factor to the rupture in the two hearts, but no rupture occurred in any of the hearts assigned to the two other groups regardless of the weight of pig or placement of the pledgets. This implies that the continuous running suture has affected the durability of the myocardial tissue. When pledgets are used in the horizontal mattress suture, the force is distributed mechanically over a greater area, while the force in the running suture seems to cut more through the annular tissue [8]. This could be a theoretical hazard in vivo since the heart is beating continuously and thereby keeps pressurizing the suture line.

The myocardial rupture in the two hearts occurred in the posterior portion of the mitral valve anulus. This supports the theory that the posterior leaflet in the 
Table 1. Individual outcome for each heart and suture method.

\begin{tabular}{|c|c|c|c|c|}
\hline \multicolumn{5}{|c|}{ Individual outcome for each heart and suture method } \\
\hline Heart (ID) & Suture method & Pressure $(\mathrm{mmHg})$ & Rupture & Weight of pig (kg) \\
\hline 1 & A & 300 & No & $40-45$ \\
\hline 2 & $\mathrm{C}$ & 300 & No & $40-45$ \\
\hline 3 & A & 300 & No & $40-45$ \\
\hline 4 & $\mathrm{C}$ & 300 & No & $40-45$ \\
\hline 5 & A & 300 & No & $40-45$ \\
\hline 6 & $\mathrm{~V}$ & 300 & No & $40-45$ \\
\hline 7 & $\mathrm{C}$ & 300 & No & $40-45$ \\
\hline 8 & A & 300 & No & $40-45$ \\
\hline 9 & $\mathrm{C}$ & 300 & No & $40-45$ \\
\hline 10 & A & 300 & No & $40-45$ \\
\hline 11 & $\mathrm{C}$ & 300 & No & $40-45$ \\
\hline 12 & $\mathrm{~V}$ & 300 & No & $40-45$ \\
\hline 13 & A & 300 & No & $40-45$ \\
\hline 14 & $\mathrm{~V}$ & 300 & No & $40-45$ \\
\hline 15 & A & 300 & No & $40-45$ \\
\hline 16 & $\mathrm{C}$ & 300 & No & $40-45$ \\
\hline 17 & A & 300 & No & $40-45$ \\
\hline 18 & $\mathrm{~V}$ & 300 & No & $25-30$ \\
\hline 19 & $\mathrm{C}$ & 300 & Yes & $25-30$ \\
\hline 20 & A & 300 & No & $25-30$ \\
\hline 21 & C & 270 & Yes & $25-30$ \\
\hline 22 & $\mathrm{C}$ & 300 & No & $40-45$ \\
\hline 23 & V & 300 & No & $40-45$ \\
\hline 24 & V & 300 & No & $40-45$ \\
\hline 25 & A & 300 & No & $40-45$ \\
\hline 26 & C & 300 & No & $40-45$ \\
\hline 27 & V & 300 & No & $40-45$ \\
\hline 28 & V & 300 & No & $40-45$ \\
\hline 29 & V & 300 & No & $40-45$ \\
\hline 30 & V & 300 & No & $40-45$ \\
\hline
\end{tabular}

A: Horizontal mattress suture with supraanular pledgets, V: Horizontal mattress suture with subanular pledgets, C: Continuous running suture.

mitral anulus is the weakest spot because the collagen fibers in the fibrotic ring are aligned towards the chordae tendinae in the posterior leaflet but circumferentially in the anterior leaflet [6] [9]. Most cardiac surgeons preserve the posterior leaflet and use it in their suture line for MVR. The above mentioned find- 
ing along with the attachment of the chordae to left ventricle and the mitral valve thereby being a part of the left ventricle function are all reasons to preserve it.

This study revealed no significant difference between the three examined suture methods though rupture was evident in two out of ten hearts sutured with the continuous running suture. This supports the findings of Newton et al. [8] and Stiles et al. [6] who suggest that sutures with pledgets are superior to non-supported sutures. We did not find any difference between subanular and supraanular pledgets as Chambers et al. [9] did.

Many factors should be considered when choosing a suture method for MVR.

The sutures with pledgets leave more foreign material within the heart than the continuous running suture, and they may require prolonged operation time. On the other hand, the horizontal mattress with pledgets placed supraannularly seem to give the best overview in explanted hearts with the atrium removed and consequently it would presumably also be applicable for MVR in human beings. With pledgets on the atrial side, it is easier for the surgeon to ensure that the pledgets are placed correctly, and that the sutures are tightened properly with even distribution of the tension.

If a patient were to get postoperative endocarditis, reoperation and removal of the former valve and sutures might be needed. In this case the horizontal mattress suture with supraanular pledgets is more beneficial since it is easier to locate the pledgets when removing the old valve. This way the surgeon can ensure that all old pledgets are removed instead of being lost in the left ventricle and thereby increasing the risk of thrombo-embolic events. The same may be applicable for ruptured sutures though supraanular pledgets from a ruptured suture could travel from the atrium to the ventricle by the PPL/PVL or through the valve itself.

The continuous running suture has no advantages in case of suture dehiscence or postoperative endocarditis since there should be an increased risk of more severe PPL/PVL as the continuous suture line would be at risk of detaching the valve. Further, a dehiscence of part of the suture line would cause the entire suture line to come undone. The latter risk, though, is controversial if the suture is handled with care. We did neither experience failure of the patch nor the suture material when testing up to $300 \mathrm{mmHg}$. Further, the Prolene 3 - 0 is a well established suture also used in aortic surgery, which is why the strength of this suture is well controlled.

A limitation of this study is that the healthy porcine hearts are obviously different from pathologically, older human hearts. In human hearts, one could expect severe calcification and fibrotic tissue which could complicate the suture placement and reduce the strength of the tissue. Therefore, one could argue that the myocardial tissue of a pathological human heart is most comparable to the young porcine hearts with less heart muscle.

The porcine heart is in general the most comparable model to a human heart, but the above-mentioned limitations apply in this study. Chambers et al. [9] and 
Newton et al. [8] investigated suture techniques in canine hearts, which are known to be more delicate, and thereby increasing the risk of rupture when applying pressure. Whichever animal model that is most comparable to a human heart with a diseased mitral valve is hard to say.

Since the investigators were not experienced heart surgeons, one could argue that it may have affected the outcome of the study, if for instance the sutures were not placed correctly in the fibrotic ring. Our findings differ from those of Chambers et al. [8], who found a mean pressure for myocardial rupture at 354 $\mathrm{mmHg}$ for horizontal mattress suture with subanular pledgets and $236 \mathrm{mmHg}$ for horizontal mattress suture with supraanular pledgets. However, the fact that we did not experience any rupture below $300 \mathrm{mmHg}$ in either of these two groups suggests that their surgical technique did not affect the outcome of this study.

In this study, it was decided that pressure above $300 \mathrm{mmHg}$ was not clinically relevant, and accordingly, we did not apply pressure until rupture.

A limitation was that we only applied pressure from the left ventricle by the means of a balloon filled with air and not liquid directly in the ventricle. Liquid could not be used because the dacron patch was oozing when filling the ventricle with water, obscuring whether the suture line was intact or not.

The shape of the balloon and the air pressure within could have had an influence on the distribution of pressure on the tissue which could differentiate it from in vivo blood pressure. We were not able to look inside the left ventricle to see the folding and shape of the balloon while applying pressure and did not apply pressure sensors inside the left ventricle to obtain pressure measurements during the testing. Furthermore, smaller leakages that could cause PPL/PVL may not be found with this model.

We cannot eliminate potential measurement errors, but we do not expect such to have had any considerable influence on the outcome.

Even though we did not find statistically significant difference between the three groups, myocardial rupture still occurred in two out of ten hearts when testing the continuous running suture. As myocardial rupture is a very serious complication and can easily result in sudden death, we cannot recommend using the continuous running suture in MVR based on our findings. Further, a larger sample size might have shown significant results, as the present result could be due to type 2 error caused by the small sample size.

Further research is needed to determine the optimal suture technique to be used in the clinic where healing, postoperative complications and the dynamics of a beating heart are taken into account.

\section{Conclusion}

At a 5\% significance level, there was no statistically significant difference between the durability of the three suture methods, though rupture was only evident in the continuous running suture group. Since it is the surgeon's choice to 
select the optimal suture technique, our study should be a reminder for the surgeon to reflect on the suture factors that have an influence on a successful MVR.

\section{Acknowledgements}

We gratefully thank the staff at the Biomedical Laboratory at the University of Southern Denmark for donating the terminated pigs and letting us use their equipment and operating room.

\section{Funding Statement}

This work was not funded.

\section{Conflicts of Interest}

The authors have declared that no competing interests exist in the work.

\section{References}

[1] Nkomo, V.T., Gardin, J.M., Skelton, T.N., Gottdiener, J.S., Scott, C.G. and Enriquez-Sarano, M. (2006) Burden of Valvular Heart Diseases: A Population-Based Study. Lancet, 368, 1005-1011. https://doi.org/10.1016/S0140-6736(06)69208-8

[2] Kim, G.J., Lee, J.T., Lee, Y.O., Cho, J.Y. and Oh, T.H. (2014) Outcomes of Nonpledgeted Horizontal Mattress Suture Technique for Mitral Valve Replacement. Korean The Journal of Thoracic and Cardiovascular Surgery, 47, 504-509. https://doi.org/10.5090/kjtcs.2014.47.6.504

[3] Kappelgaard, Lv., Toftlund, S.A., Davidsen, M. and Gislason, G. (2020) Dansk Hjerteregister-Årsberetning 2019.

[4] Ionescu, A., Fraser, A.G. and Butchart, E.G. (2003) Prevalence and Clinical Significance of Incidental Paraprosthetic Valvar Regurgitation: A Prospective Study Using Transoesophageal Echocardiography. Heart, 89, 1316-1321. https://doi.org/10.1136/heart.89.11.1316

[5] Dhasmana, J.P., Blackstone, E.H., Kirklin, J.W. and Kouchoukos, N.T. (1983) Factors Associated with Periprosthetic Leakage Following Primary Mitral Valve Replacement: With Special Consideration of the Suture Technique. The Annals of Thoracic Surgery, 35, 170-178. https://doi.org/10.1016/S0003-4975(10)61456-7

[6] Stiles, G.M., Kernen, J.A. and Stiles, Q.R. (1986) Suture Technique in Preventing Dehiscence of Prosthetic Mitral Valves. The Archives of Surgery 121, 1136-1140. https://doi.org/10.1001/archsurg.1986.01400100042008

[7] Azam, H., Hussain, G., Ahmed, N., Raza Baig, M.A., Zaheer, S. and Ali Gilani, S.R. (2015) Comparison of Semi-Continuous and Interrupted Suture Techniques for Mitral Valve Replacement. Journal of Pakistan Medical Association, 65, 844-846.

[8] Newton Jr., J.R., Glower, D.D., Davis, J.W. and Rankin, J.S. (1984) Evaluation of Suture Techniques for Mitral Valve Replacement. The Journal of Thoracic and Cardiovascular Surgery, 88, 248-252. https://doi.org/10.1016/S0022-5223(19)38359-X

[9] Chambers Jr., E.P. and Heath, B.J. (1991) Comparison of Supraannular and Subannular Pledgeted Sutures in Mitral Valve Replacement. The Annals of Thoracic Surgery, 51, 60-63. https://doi.org/10.1016/0003-4975(91)90450-5 